\title{
Implementation of Data Synchronization Mechanism in Virtual Desktop
}

\section{Infrastructure}

\author{
Chiao-Tung Yang ${ }^{1, *}$, Jheng-Yue $\mathrm{Li}^{2}$, Cai-Jin Chen ${ }^{3}$, Chun-I Chiang ${ }^{4}$ \\ Dept. of Computer Science, Tunghai University, Taiwan \\ ${ }^{1}$ ct.yang@thu.edu.tw*; ${ }^{2}$ as881028@gmail.com; ${ }^{3}$ amranchen@yahoo.com; ${ }^{4}$ jerry1526@yahoo.com.tw \\ * corresponding author
}

(Received: August 8, 2021; Revised: August 22, 2021; Accepted: October 12, 2021; Available online: December 1, 2021)

\begin{abstract}
There are more and more applications and services of Cloud technology. Whether the government, enterprises or organizations, even everyone may have requirements of constructing cloud systems. To build the virtual cloud infrastructure VMware vSphere which is the leader of the information industry on virtual platforms can let users use Virtual Desktop Infrastructure by their mobile device. This study integrated some management programs such as Activity Directory, VMware vCenter, View connection server, VMware vSphere and used the shared storage system of FreeNAS to provide the cloud virtual environment to users. With the simple interface to manage, it successfully provides administrators to build Virtual Desktop Infrastructure faster. Users can use their own device to control the virtual desktop in a mobile classroom by VMware Horizon View or browser. Users can also use a shared storage system to be the information exchange platform. If you can connect to the Internet, you can use your own virtual desktop anywhere. Finally, the complete construction implements the combination of several operating systems and improves their performance to save lots of hardware cost. It also meets the social focus of carbon reduction and environment protection to achieve sustainable use.
\end{abstract}

Keywords: VMware vSphere; VMware vCenter; FreeNAS; VMware Horizon

\section{Introduction}

Nowadays, to provide certain workers with the infrastructure for a virtual rather than physical computer is possible for many reasons, including increased security [1], lower cost, taking up less space, and easier management system. So, the first step of architecture development is more important to the next operation [2].

After reviewing several times, we found out one of the virtual devices that has this power is Virtual Desktop Infrastructure [3]. Virtual Desktop Infrastructure has several benefits as bellow:

- Security

Security is the main reason for rolling out VDI. In VDI, you have a good control to lock the image from external devices. It means your image data cannot be copied to a local machine. All of the information will be protected in the system.

- Management

On the management side, if we have a stand-alone desktop to manage hundreds of desktops, it is really not easy to do. So, Virtual Desktop Infrastructure (VDI) allows you to have a centralized management of all your desktops and control what is being installed and run on a hundred desktop environments. 
- Operating System migration

With a hundred desktop environments, if you want to upgrade one by one desktop application it needs a long time period to finish this progress. But with centralized management of VDI, you can carry out a new operating system from a central location to the group environment.

- Snapshot function

In the Virtual Desktop Infrastructure environment, you have an ability to roll back your desktops to their previous state [4]. It gives a lot of flexibility in usage. Here, this study uses Virtual Desktop Infrastructure (VDI) to build a new environment in order for users to access shared storage systems [5]. This shared storage system is called FreeNAS.

\section{Background and Related Work}

In this section, this study will explain some backgrounds and related works which are reviewed after our experiment.

\subsection{Virtual Desktop Infrastructure}

Based on Huang et al., [6] Raspberry Pi is an open source hardware project platform with a circuit board which has simple I/O connector function and a number of Linux software usage. Raspberry Pi can be used for interactive product development. As an example, it can be used to read a large number of switches or sensor signals and also can be used to control lamps, electrical and any other physical devices [7]. Raspberry Pi is also used on personal computers (PC) that have the same peripheral devices, it also can communicate with Linux operating systems.

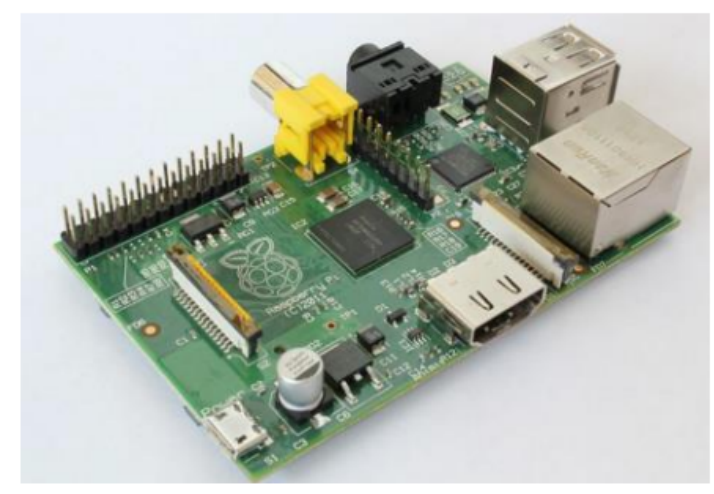

Fig. 1. Raspberry Pi tool

Beside Raspberry Pi as a tool to access the system, the other device that can access to VDI is Wandboard. Wandboard is a low cost device rich Cortex-A9 board based on the NXP i.MX6 family of SoCs. The following is a Wandboard device.

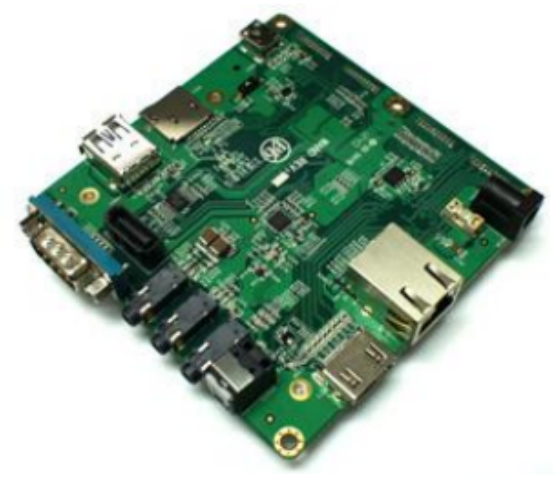

Fig. 2. Wandboard tool

Wang et al., [8] define virtual desktop infrastructure as a software technology that uses desktop environments and related applications to read and store data from client device separation. Virtual Desktop Infrastructure can be used in 
conjunction with virtualization applications and user profile management systems [9]. Now, it is known as the "User virtualization". It provides a comprehensive desktop management system environment. In this mode, all substantial elements in VDI are virtualized.

This allows a highly flexible and safe virtual desktop environment. In addition, this approach supports more disaster recovery strategies [10]. Because all substantial elements are stored in the other devices through the maintenance and backup system. If the user devices or hardware are lost, the recovery is very simple, basically all desktop components may be registered in the other devices [11]. Otherwise, because of data unavailable. If the device is lost, and it will be able to reduce its loss, what happened in this condition the data should be returned. The following is more detail of the type to be used in virtual desktop technology deployment.

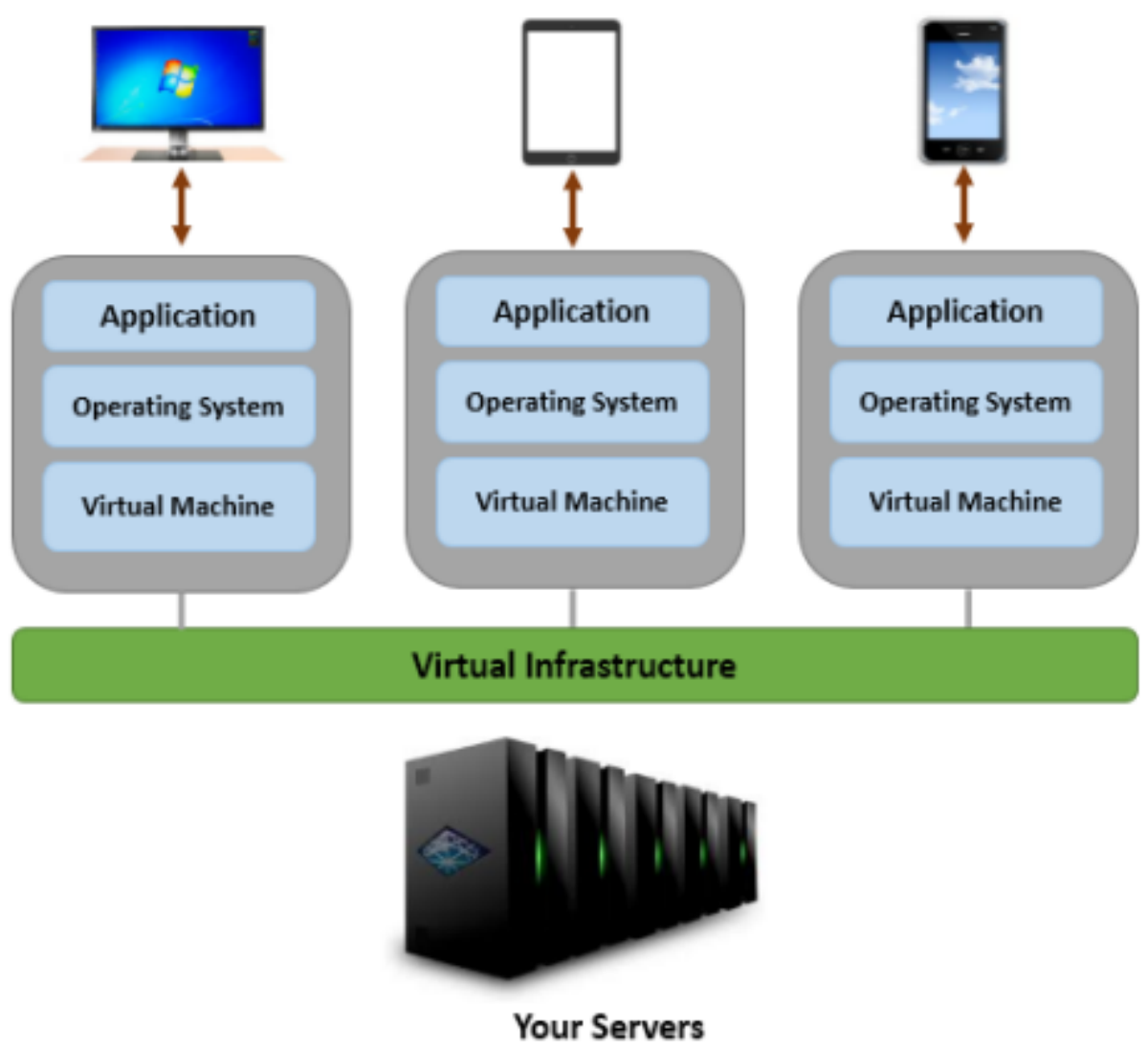

Fig. 3. Basic Virtual Desktop Infrastructure

\subsection{Cloud Storage}

Bhako [12] stated that cloud storage is an online storage mode where data is stored on third-party multiple virtual servers rather than on a dedicated server. Hosting companies operate a large data center for people who need the data storage. Then through their purchase or lease of the storage space, they find the data storage demand. Data center serves customer demand, the rear end prepares to provide a virtualized storage pool [13]. Users will use the storage pool to store their data. Indeed, these resources may be distributed over a number of host servers. Figure 4 shows a rough sketch of the centralized shared storage system of FreeNAS that you can access anywhere at any time. 


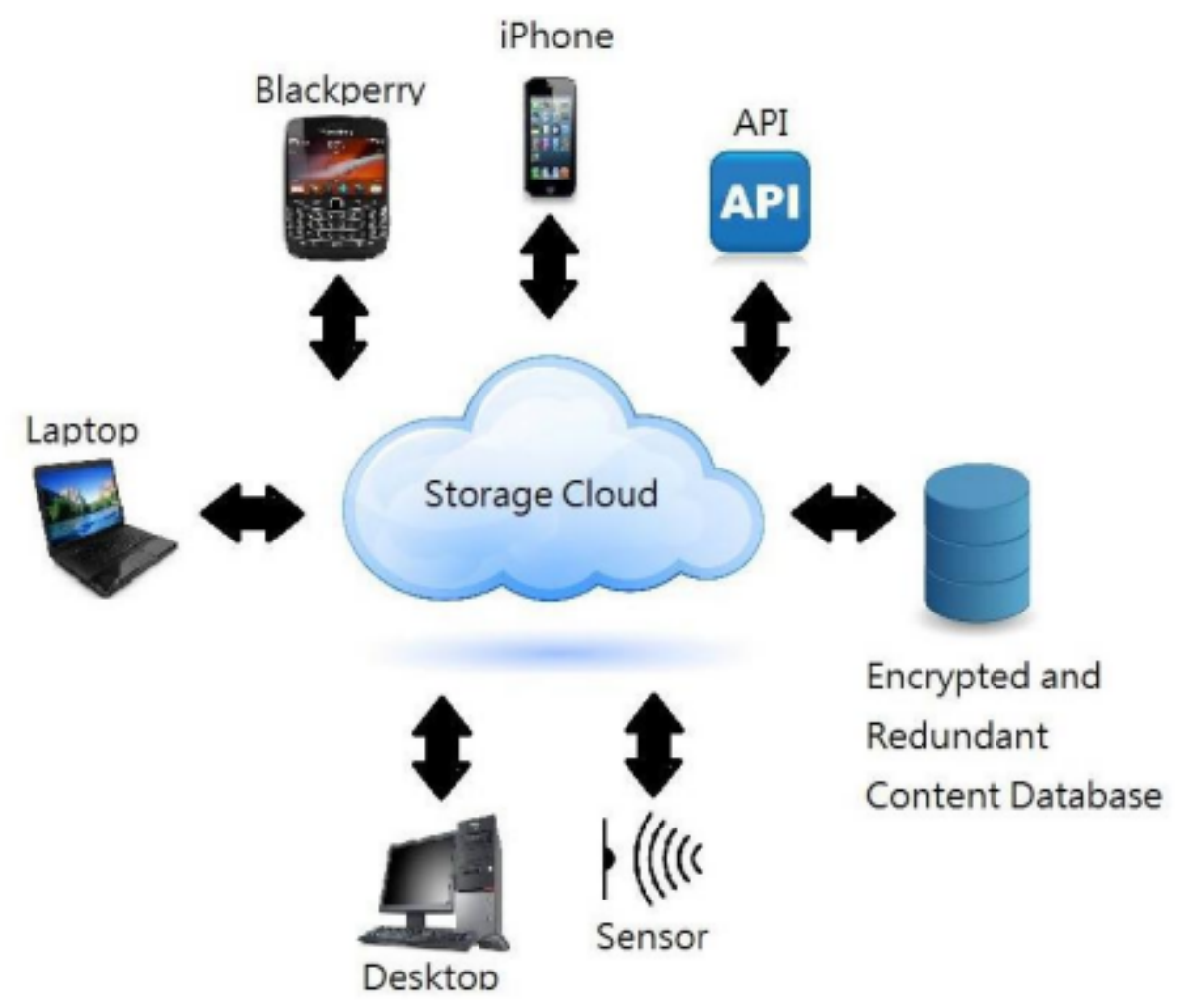

Fig. 4. Cloud Storage environment

\subsection{Network Attached Storage}

Based on Rasna [14], network Attached Storage is a special name of data storage technology, it can be connected to the computer network environment directly. For the heterogeneous network, the user provides the centralized data access services. NAS allows increased data usage. The main reason is the data does not depend on the servers. Users cannot use the data because of the server service shutdown [15], it is already linked by NAS storage. NAS storage also makes data management easier and simpler, so the original needs to process the complicated formula on the server, simplified into a few steps that can be completed, greatly to save time. The NAS device operating system only contributes data storage, data access, and related management functions [16]. In addition, NAS devices also offer more than one file transfer. NAS systems typically have more than one hard disk, and the same with traditional file transfer, they will usually be composed of RAID to provide services. With the next NAS device system, other servers on the network can no longer serve as file server functions [17]. NAS has a diversity form, can be a mass-produced embedded device, you can also perform NAS software on a general computer. NAS uses file based protocols such as NFS or SMB. In contrast to SAN, SAN is based on a block of coprocessor units, ordinary through SCSI switch to Fiber Channel or iSCSI. NAS storage or device usage generally uses a streamlined version of the operating system, only contributing the most simple file and related communication agreement [18]. For example, there is an open source NAS software that is called as FreeBSD Lite, it can be run on a computer, the embedded devices commercial version use commercial operating system and communication protocol application

\subsection{Related Work}

With the global economy to get more competitive, users always look for a platform that can respond faster to changing faster working solutions [19], cost saving and ensure continual growth. For alternative solutions that arrest the proliferation of desktops, minimize desktop management time and reduce operational expenses. Virtual Desktop Infrastructure (VDI) solutions to create a centrally-managed virtual desktop environment that streamlines the planning, rollout and management of desktop infrastructure [11]. It runs desktop operating systems and application software inside a virtual machine. Users access these virtual desktops using their existing PCs. This not only eliminates the need for workstation hardware upgrades, but also enables the user to switch between operating environments, such as Windows XP and Windows 7. 
The first benefit of the VDI system is that the desktop can use the same image. The operating system as well as applications that are installed, decreases organizational and support costs. Images will need to be tested as new applications are introduced, and either additional images will need to be created or adjustments will have to be made to the current build. Also, depending on your model, if you use persistent desktops, incremental storage space will be needed for VDI servers since user needs vary, i.e. saving private settings if users are allowed to install their own applications.

While an investment in server hardware and storage infrastructure may be required, keep in mind that everything will be hosted in the data center, therefore, there may not be a need for a huge collection of standard PCs in the office.

\section{System Design and Implementation}

The experimental system design is very important to build a new system. So, current research needs to analyze this system before experiment. For more detail of the experimental system, this study can see further experiment description. They consist of two parts: experimental environment and system architecture.

\subsection{Experimental environment}

Our Raspberry Pi device for experimental purposes consists of five plugs that all use USB type. The following is five kind of plugs that used:

- Keyboard plug

This plug is used to connect the keyboard to Raspberry Pi.

- Mouse plug

This mouse plug is used to connect our mouse to Raspberry Pi.

- Network cable plug

In order our Raspberry Pi can remote to the Virtual Desktop Infrastructure environment, we need internet connection usage.

- Power connector plug

To have the power supply, it needs a USB cable plug to connect our Raspberry Pi to the socket.

- Monitor cable plug

Monitor becomes an important thing in computer components. Without a monitor, we cannot show anything on the layer. So, this study also needs one plug for this device.

Figure 5 shows us the Raspberry Pi device that consists of five plugs.
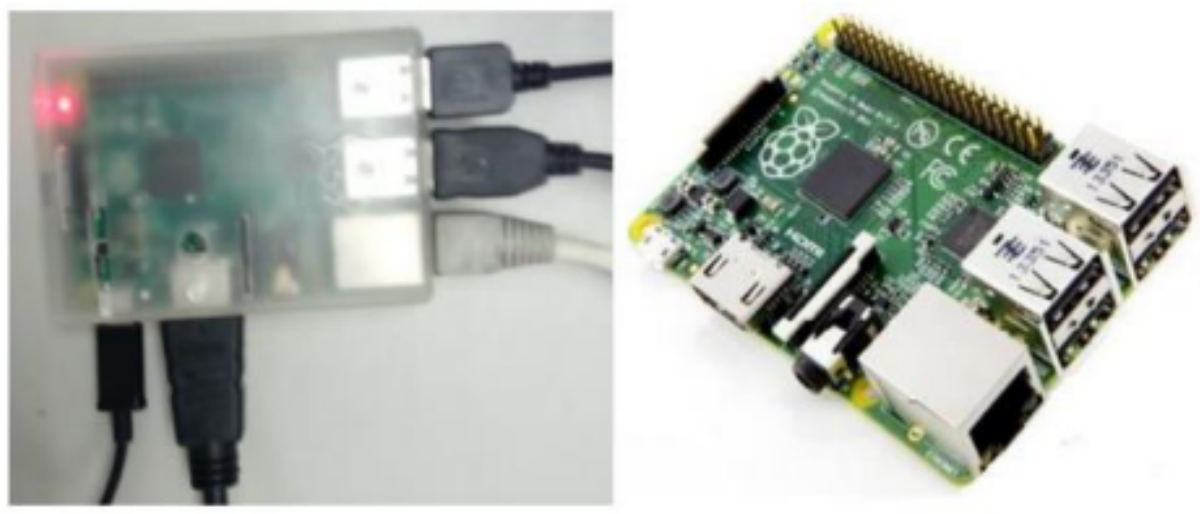

Fig. 5. Raspberry Pi device

Besides Raspberry Pi as a tool to access the Virtual Desktop Infrastructure environment, we can use Wandboard tool to access the Virtual Desktop Infrastructure too. Figure 6 shows the device. 
Yang et al / IJIIS vol. 4, no. 3, December 2021, pp 157-167

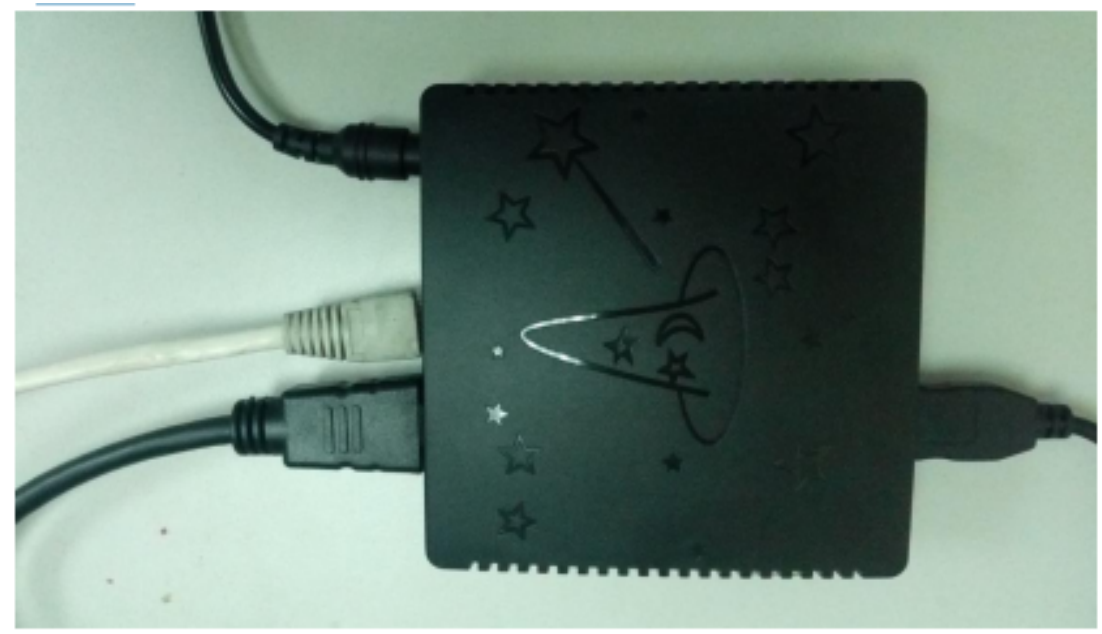

Fig. 6. Wandboard device

Below is the wandboard environment on the monitor.

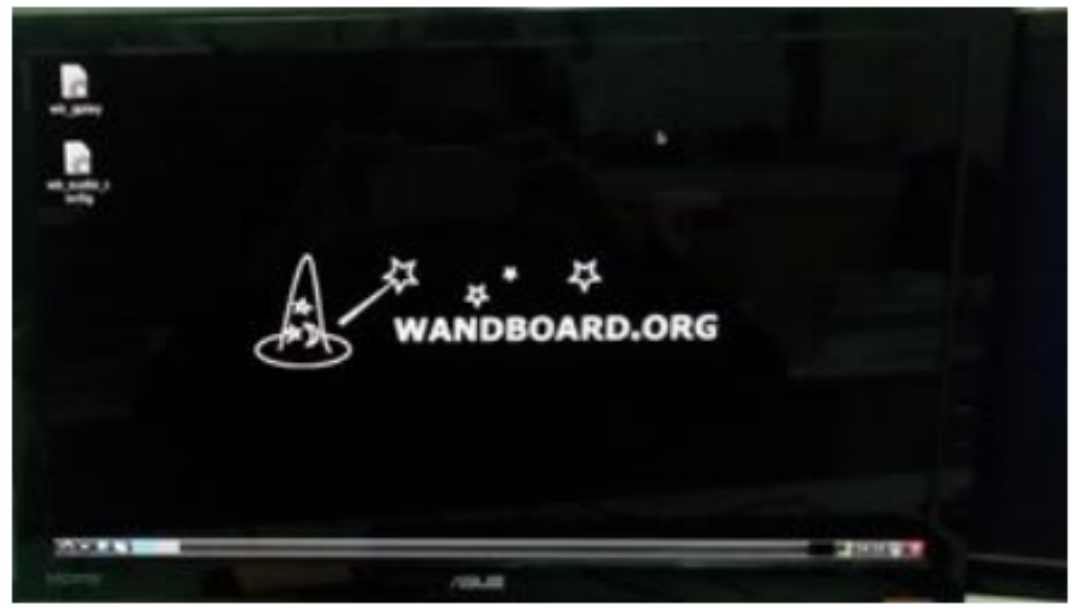

Fig. 7. Wandboard Environment

Physical server is the main device to develop Virtual Desktop Infrastructure and share storage with FreeNAS. Here is the condition of our physical server that is used for implementation.

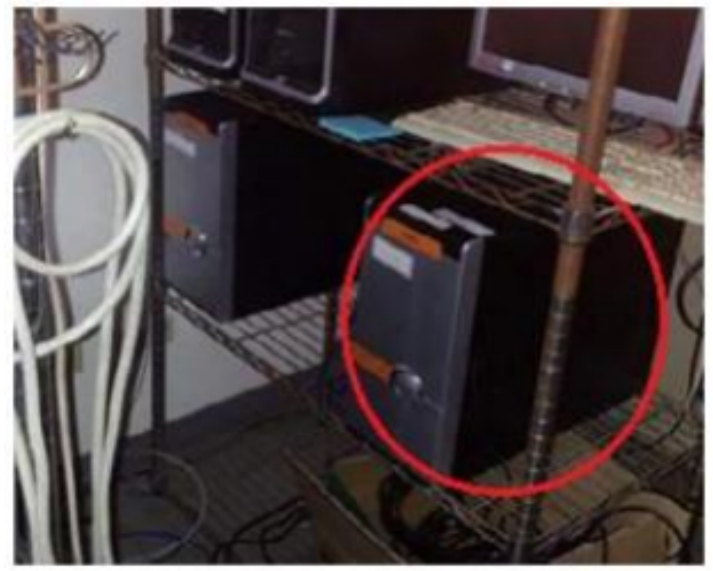

Fig. 8. FreeNAS server 


\subsection{System Architecture}

Figure 9 shows our experimental system architecture. This architecture consists of four sets of Virtual Desktop Infrastructures, one set FreeNAS, Raspberry Pi and VMWare Horizontal client software as a platform that is used for accessing our Virtual Desktop Infrastructure environment.

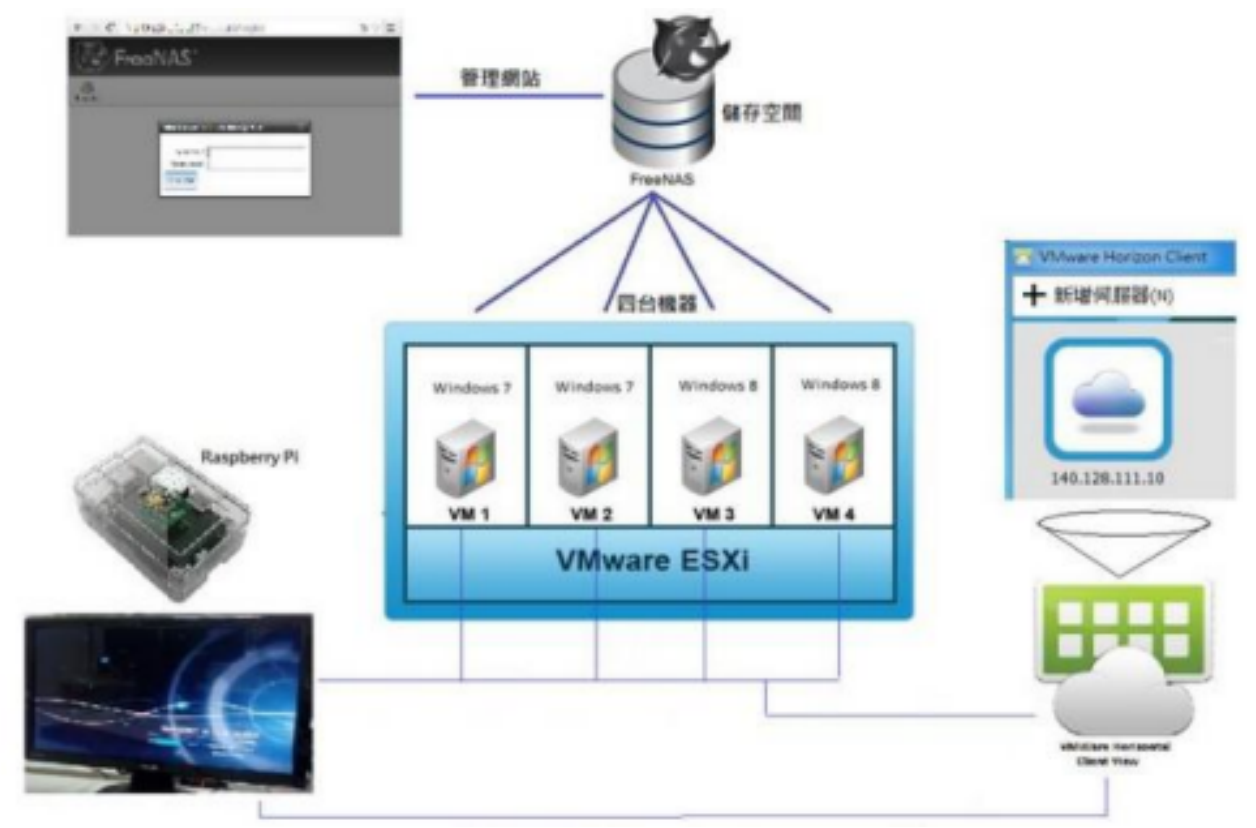

Fig. 9. VDI Environment and NAS storage architecture

Workflow process description in this study describes this workflow as a user position to use this system. This user uses Raspberry Pi or VMWare Horizontal Client View logs into the Virtual Desktop environment. The first step that the user has to do is enter the Virtual Desktop Infrastructure IP Address with an account. After logging in, users already enter the Virtual Desktop Infrastructure environment. This Virtual Desktop Infrastructure environment has been linked to FreeNAS shared-storage drive to access the data if available. If the data is still unavailable, we can create or add new data. As long as the user has the internet connection, the user is ready to connect this Virtual Desktop Infrastructure environment and shared storage system of FreeNAS. To remote the Virtual Desktop Infrastructure environment, users can use VMWare Horizontal client view tool.

\section{Experimental Results}

\subsection{Experimental Environment}

In this section, this study describes our data synchronization mechanism in the Virtual Desktop Infrastructure (VDI) system. Table 1 lists the hardware specification of the Virtual Desktop Infrastructure servers in the experiment. This study uses four servers for VDI and one server for FreeNAS storage.

Table. 1. Virtual Desktop Infrastructure server

\begin{tabular}{|l|l|}
\hline Specification & Description \\
\hline Processor & Intel Xeon E5540@2.53GHz \\
\hline RAM & $8 \mathrm{~GB}$ \\
\hline Operating System & Windows 7 SP1, Windows 8 SP1 \\
\hline Computer Name & $\begin{array}{l}\text { CT-STUDENTS-1, CT-STUDENTs-2 CT-WIN8-1, } \\
\text { CT-WIN8-2 }\end{array}$ \\
\hline Domain & vcrs di.local \\
\hline
\end{tabular}


Yang et al / IJIIS vol. 4, no. 3, December 2021, pp 157-167

\begin{tabular}{|l|l|}
\hline Hard disk size & 80 GB per server \\
\hline
\end{tabular}

Table. 2. FreeNAS specification

\begin{tabular}{|l|l|}
\hline Specification & Description \\
\hline Processor & Intel Xeon E5540@2.53GHz \\
\hline RAM & 8 GB \\
\hline Operating System & FreeNAS 0.98 version \\
\hline Hard disk Type & 2 set hard disks with RAID 10 \\
\hline Hard disk Size & $100 \mathrm{~GB}$ \\
\hline
\end{tabular}

\subsection{Experimental Results}

This figure 10 shows the storage management interface that users can get through anywhere and anytime to open up a web browser to visit the FreeNAS management interface. Just visit IP Address and enter user account, user can manage shared-storage. Here, besides starting/stop the FreeNAS service, user also can add user account, set up user level privileges or grant access, add new storage, starts the other environment services beside NFS service and so on.

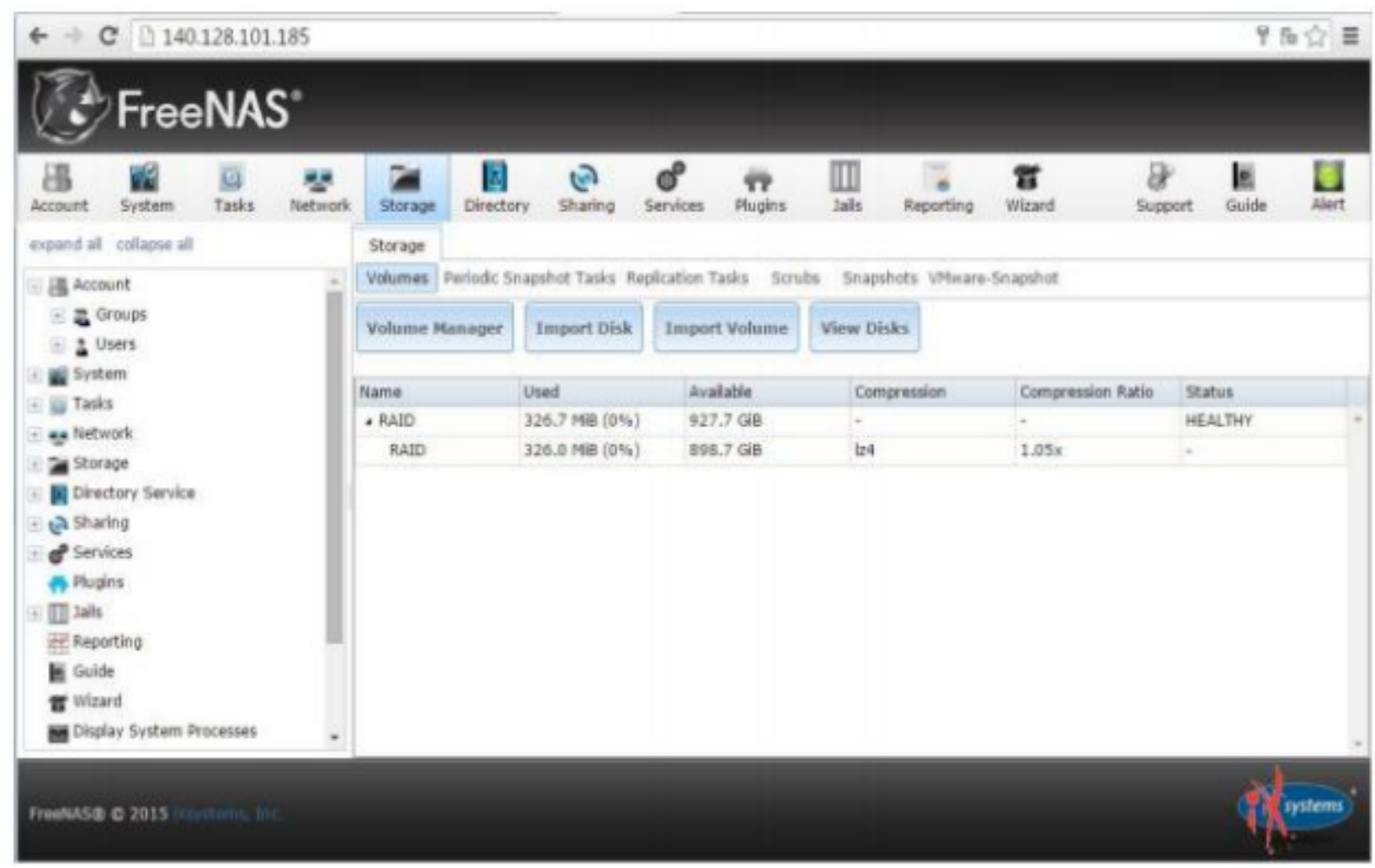

Fig. 10. FreeNAS management interface

After logging into the Virtual Desktop Infrastructure environment by a user account, you can access the shared storage system of FreeNAS to add, update, delete some documents or data, even create a folder to group your documents or data [20]. Keep in mind when a user accesses this shared storage system of FreeNAS, the user has to enter a freeNAS user account that was created on the FreeNAS management website for the second time after 
Yang et al / IJIIS vol. 4, no. 3, December 2021, pp 157-167

logging into the Virtual Desktop Infrastructure environment [21]. This procedure is carried out in order to make user data more secure. The following figure 11 is the log-in FreeNAS drive environment.

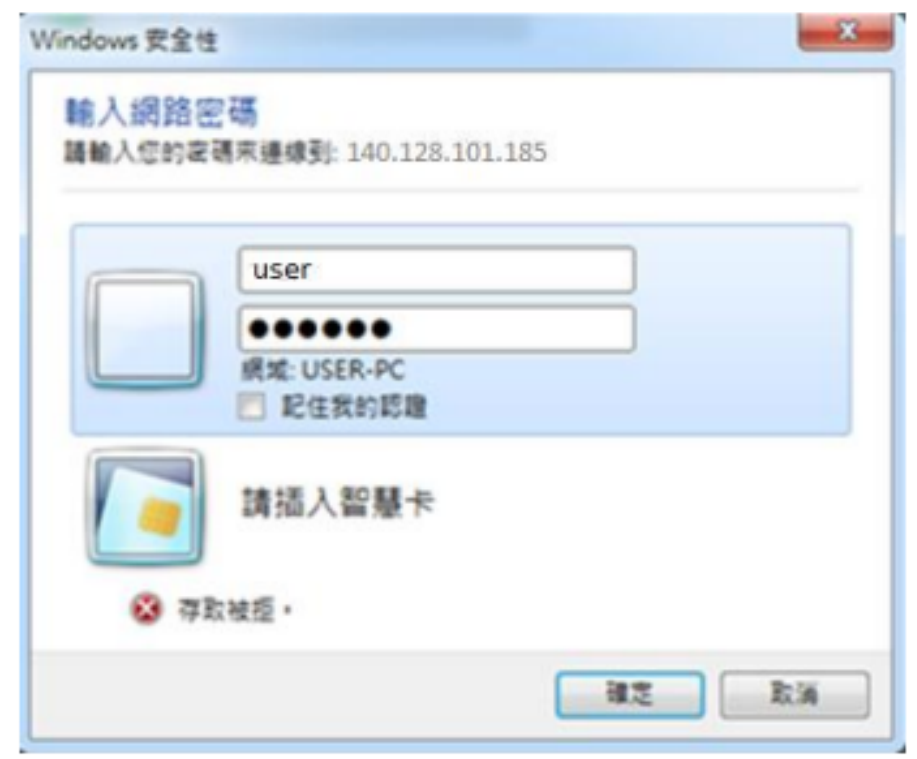

Fig. 11. FreeNAS drive authentication environment

Figure 12 shows a shared storage system of FreeNAS that users can access anywhere and anytime at least the user has an internet connection to the remote.

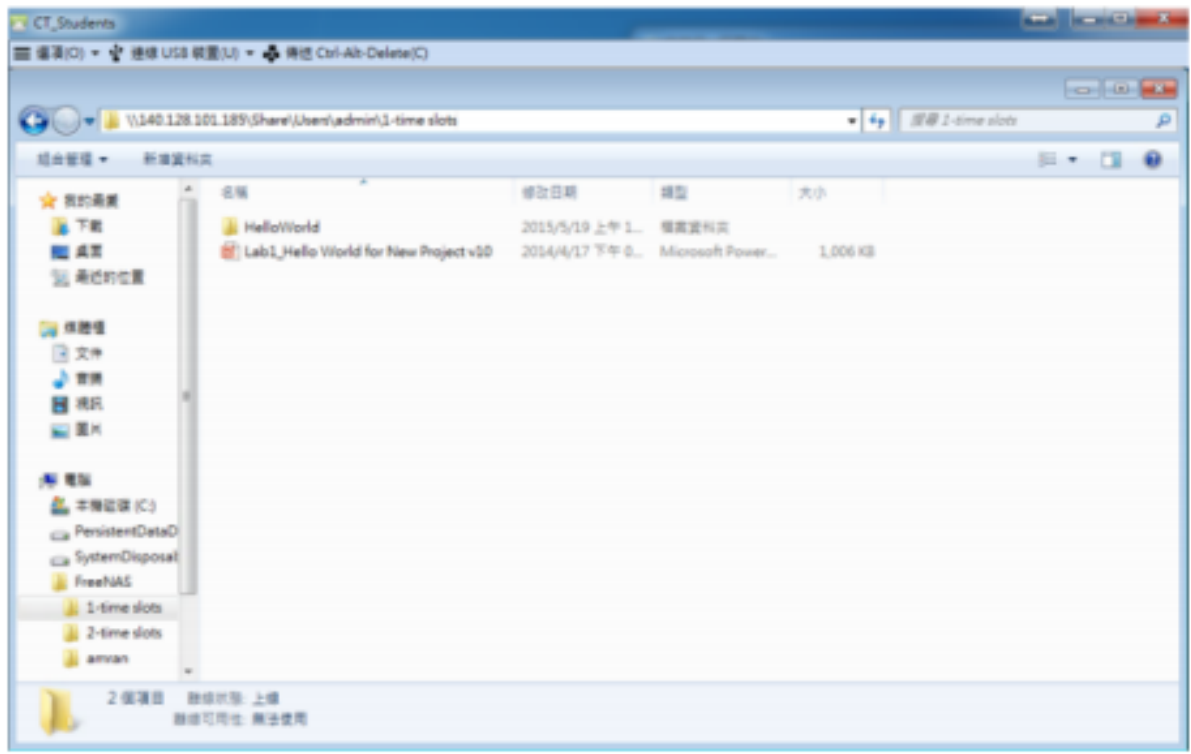

Fig. 12. Shared storage system of FreeNAS 


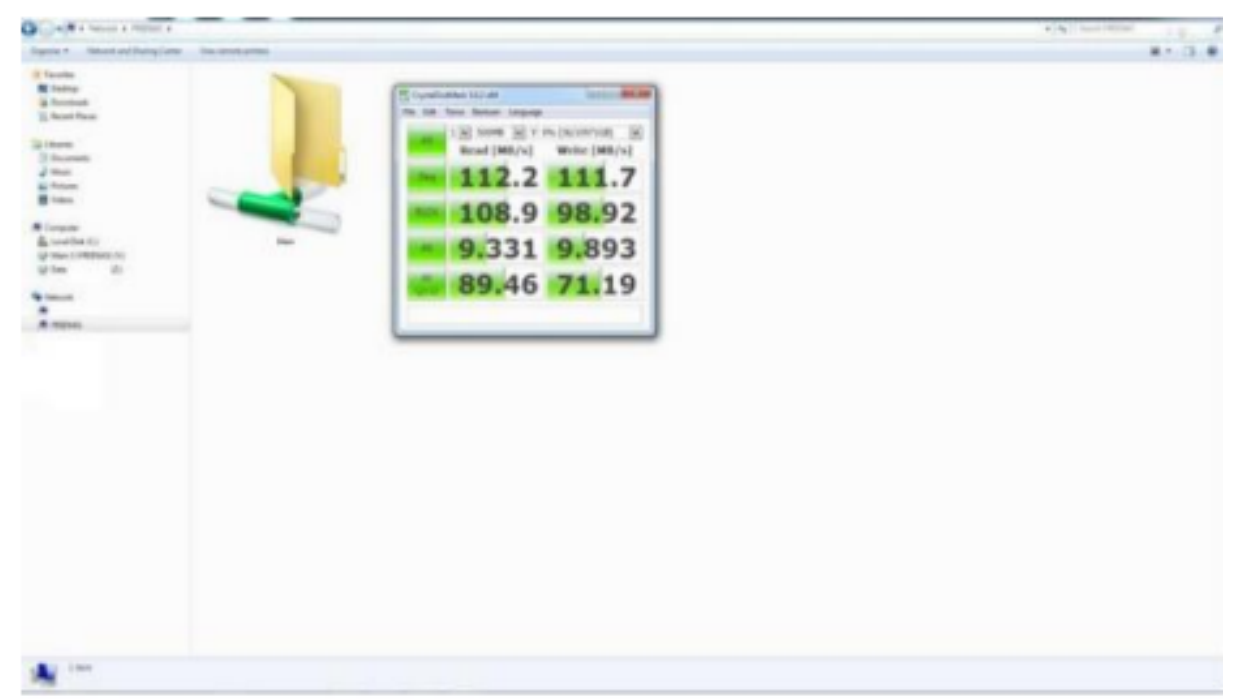

Fig. 13. Access speed test result

\section{Conclusions and Future Work}

\subsection{Conclusions}

During the experiment conducted, this study can make a conclusion for all activities performed. With integrate some management programs such as Activity Directory, VMware vCenter, View connection server, VMware vSphere and used the shared storage system of FreeNAS to provide the cloud virtual environment to user, this Virtual Desktop Infrastructure environment make easier for user to do their jobs at anywhere on anytime without provide more storage at their place. The main point is users must have an internet connection and some devices, for example Raspberry Pi or Wandboard, Monitor, Keyboard, Mouse and so on to access the Virtual Desktop Infrastructure servers to do their job in the shared storage system of FreeNAS. Besides that, this shared storage can be adjusted for user level privileges or grant access. With this system, users already reduce their cost saving device and place.

\subsection{Future Work}

In the future, this experiment can be developed not only in the Windows environment to access the shared storage system of the FreeNAS environment, it also can be carried for the other operating system environment, for example Linux operating system, Mac OS and so on. The other consideration, we can build other shared-storage systems and we can compare with this shared storage system of FreeNAS to know each access speed software, the advantages and disadvantages of both or more of these softwares.

\section{References}

[1] H. Zhou, S. Ma, and W. Li, "Actuator saturating intermittent control for synchronization of stochastic multi-links network with sampled-data," Neurocomputing, vol. 465, pp. 167-183, 2021, doi: 10.1016/j.neucom.2021.08.123.

[2] K. J. Co, A. V. Ong, and M. Peradilla, "WSN Data Collection and Routing Protocol with Time Synchronization in Low-cost IoT Environment," Procedia Comput. Sci., vol. 191, no. 2019, pp. 102-110, 2021, doi: 10.1016/j.procs.2021.07.016.

[3] R. Zhang, X. Song, Y. Zhang, and S. Song, "Dissipative sampled-data synchronization for spatiotemporal complex dynamical networks with semi-Markovian switching topologies," Neurocomputing, vol. 448, pp. 333-343, 2021, doi: 10.1016/j.neucom.2021.03.086.

[4] L. Lin, J. Zhong, S. Zhu, and J. Lu, "Sampled-data general partial synchronization of Boolean control networks," J. Franklin Inst., vol. 3, no. 3, pp. 1-11, 2020, doi: 10.1016/j.jfranklin.2020.08.047. 
Yang et al / IJIIS vol. 4, no. 3, December 2021, pp 157-167

[5] P. Nirvin, F. A. Rihan, R. Rakkiyappan, and C. Pradeep, "Impulsive sampled-data controller design for synchronization of delayed T-S fuzzy Hindmarsh-Rose neuron model,” Math. Comput. Simul., no. 3, pp. 23-44, 2021, doi: 10.1016/j.matcom.2021.03.022.

[6] Z. Huang, L. Chen, L. Zhang, S. Fan, and D. Fan, "Research on software synchronization method of real-time ethernet distributed motion control system," Assem. Autom., vol. 39, no. 5, pp. 904-916, 2019, doi: 10.1108/AA-12-2018-0265.

[7] D. Maillard, “The Obsolescence of Man in The Digital Society,” Int. J. Appl. Inf. Manag., vol. 1, no. 3, pp. 99-124, 2021, doi: 10.47738/ijaim.v1i3.13.

[8] Y. Wang, J. Guo, G. Liu, J. Lu, and F. Li, "Finite-time sampled-data synchronization for uncertain neutral-type semi-Markovian jump neural networks with mixed time-varying delays," Appl. Math. Comput., vol. 403, p. 126197, 2021, doi: 10.1016/j.amc.2021.126197.

[9] B. S. Larkin, "Increasing Information Integrity: Cultural Impacts of Changing The Way We Manage Data," Eletronic Libr., vol. 34, no. 1, pp. 1-5, 2018.

[10] C. Augustine, "The synchronization of business cycles in CARICOM: Is there a case for a Caribbean Monetary Union?,” Int. J. Dev. Issues, vol. 7, no. 1, pp. 29-40, 2008, doi: 10.1108/14468950810880008.

[11] J. Woznica and K. Healy, "The level of information systems integration in SMEs in Irish manufacturing sector," J. Small Bus. Enterp. Dev., vol. 16, no. 1, pp. 115-130, 2009, doi: 10.1108/14626000910932917.

[12] V. Bhakoo and C. Chan, "Collaborative implementation of e-business processes within the health-care supply chain: The Monash Pharmacy Project," Supply Chain Manag., vol. 16, no. 3, pp. 184-193, 2011, doi: $10.1108 / 13598541111127173$.

[13]M. Jeffery, R. J. Sweeney, and R. J. Davis, "Teradata Data Mart Consolidation Return on Investment at GST," Kellogg Sch. Manag. Cases, vol. 3, pp. 1-20, 2017, doi: 10.1108/case.kellogg.2016.000337.

[14]R. Rasna, "Comparison of Security Signing Data Authentication Integrity in Combination of Digest And AES Message Algorithm,” IJIIS Int. J. Informatics Inf. Syst., vol. 4, no. 1, pp. 1-12, 2021, doi: 10.47738/ijiis.v4i1.72.

[15] A. Faizah, "Implementation of the Convolutional Neural Network Method to Detect the Use of Masks," IJIIS Int. J. Informatics Inf. Syst., vol. 4, no. 1, pp. 30-37, 2021, doi: 10.47738/ijiis.v4i1.75.

[16]C. Ma, D. C. Chou, and D. C. Yen, "Data warehousing, technology assessment and management," Ind. Manag. Data Syst., vol. 100, no. 3, pp. 125-134, 2000, doi: 10.1108/02635570010323193.

[17] A. S. M. Moohialdin, F. Lamari, M. Miska, and B. Trigunarsyah, "Construction worker productivity in hot and humid weather conditions: A review of measurement methods at task, crew and project levels," Eng. Constr. Archit. Manag., vol. 27, no. 1, pp. 83-108, 2020, doi: 10.1108/ECAM-05-2018-0191.

[18] S. Li, J. K. Visich, B. M. Khumawala, and C. Zhang, "Radio frequency identification technology: Applications, technical challenges and strategies," Sens. Rev., vol. 26, no. 3, pp. 193-202, 2006, doi: 10.1108/02602280610675474,

[19]H. Hitoshi, "The Effectiveness of The Body of Knowledge Process in The Startup Analysis of Efficiency by Applying Startup Management Body of Knowledge (SUBOK) Guide,” Int. J. Appl. Inf. Manag., vol. 1, no. 2, pp. 28-49, 2021, doi: 10.47738/ijaim.v1i2.11.

[20]J. Yeung, "Data Analytics Architectures for E-Commerce Platforms in Cloud," Int. J. Appl. Inf. Manag., vol. 1, no. 1, pp. 1-5, 2021, doi: 10.47738/ijaim.v1i1.3. 